\title{
Citogenética do gênero Leucaena Benth
}

\author{
Cytogenetics of the genus Leucaena Benth
}

\author{
Maria Teresa Schifino-Wittmann ${ }^{1}$
}

- REVISÃO BILBLIOGRÁFICA -

RESUMO

Neste trabalho, são revistos e discutidos os resultados mais relevantes dos estudos citogenéticos em Leucaena realizados pelo grupo do Departamento de Plantas Forrageiras e Agrometeorologia da Universidade Federal do Rio Grande do Sul, que envolvem híbridos selecionados dentro de programas de melhoramento, e as espécies selvagens e cultivadas do gênero. Estes resultados revelaram variabilidade intra e interespecífica para o número cromossomico, mostrando a ocorrência de multivalentes mesmo nas espécies consideradas diplóides (o que apóia a origem paleopoliplóide de muitas espécies) e constituem uma importante contribuição para a citogenética das espécies de Leucaena, com repercussões na taxonomia e no melhor entendimento da complexa evolução do gênero. Os resultados indicam que deve haver um acompanhamento citogenético em programas de melhoramento genético destas espécies.

Palavras-chave: citogenética, evolução, Leucaena, melhoramento.

\section{ABSTRACT}

The cytogenetics group of the Departamento de Plantas Forrageiras e Agrometeorologia, Universidade Federal do Rio Grande do Sul, Brazil determined the chromosome numbers of all species of Leucaena. Cytogenetic results of some selected hybrids and wild and cultivated species of Leucaena are reviewed and discussed. The results showed an intra and interespecific chromosome number variability and the occurrence of multivalents even in the presumed diploid species (what supports a paleopolyploid origin for these species). This is a very important contribution and may reflect on the taxonomy and on the understanding of the complex evolution of Leucaena. These results suggest that cytogenetic studies should be part of all Leucaena breeding programs.

Key words: cytogenetics, evolution, Leucaena, plant breeding.

\section{INTRODUÇÃO}

O gênero Leucaena (Mimosae, Mimosoideae, Leguminosae) compreende árvores (Figura 1) fixadoras de nitrogênio, nativas das Américas e distribuídas desde o sul do Texas até o Perú. As múltiplas utilizações destas árvores incluem a produção de madeira, alimentação humana, forragem para o gado, adubação verde, sistemas agroflorestais, artesanato, cercas e cercas vivas, etc. Além de economicamente importantes em algumas de suas utilizações específicas, como forragem e produção de madeira, o fato de serem árvores multipropósito faz com que muitas destas espécies sejam amplamente utilizadas em locais mais pobres das regiões tropicais. Espécies como L. leucocephala Lam. podem comportar-se como invasoras agressivas (HUGHES, 1993). Algumas espécies, principalmente $\boldsymbol{L}$. leucocephala e L. diversifolia Schltdl, e em menor escala $L$. pallida Britton \& Rose, são amplamente cultivadas fora de sua região de origem, especialmente nos trópicos (HUGHES, 1993; HUGHES, 1998a; HUGHES, 1998b). Em regiões de clima temperado a subtropical, como, por exemplo, no sul do Brasil, tem havido muito interesse na utilização de Leucaena como uma alternativa forrageira, ou em sistemas agroflorestais (SCHIFINO-WITTMANN, 2000).

A taxonomia do gênero tem sido discutida por vários autores (BREWBAKER, 1987; HUGHES, 1993; ZÁRATE, 1994). Recentemente, uma ampla revisão taxonômica envolvendo morfologia, análise

${ }^{1}$ Biólogo, Professor Adjunto, Doutor, Departamento de Plantas Forrageiras e Agrometeorologia, Faculdade de Agronomia, Universidade Federal do Rio Grande do Sul. CP 15100, 91501-970, Porto Alegre, RS, Brasil. Bolsista do CNPq. E-mail: mtschif@ ufrgs.br. 


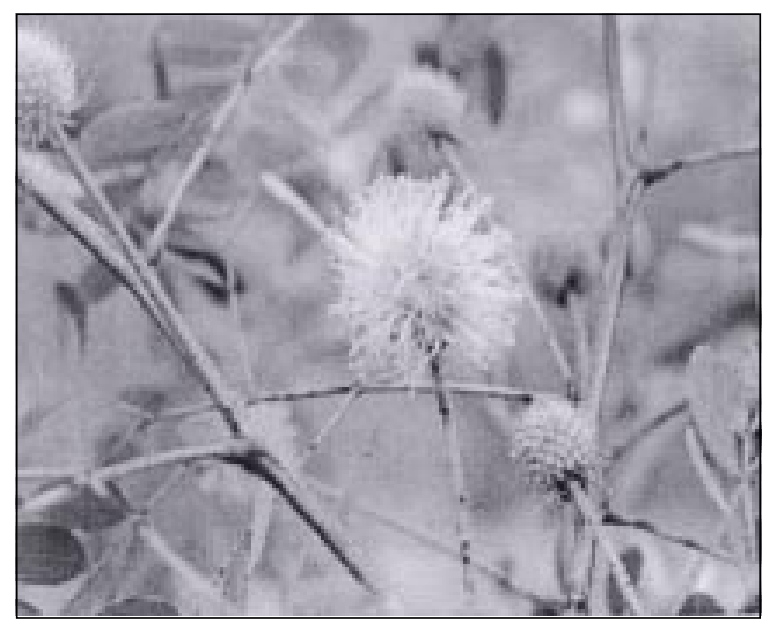

Figura 1 - Inflorescência de L. retusa

cladística e dados de cpDNA (HUGHES, 1998a) delimitou 22 espécies, quatro subespécies, duas variedades e dois taxa híbridos. A evolução do gênero parece ser bastante complexa, envolvendo especiação reticulada, e é sugerida uma ação antrópica no surgimento de L. leucocephala (HUGHES \& HARRIS, 1995; HARRIS et al., 1996).

Os cromossomos de Leucaena são pequenos $(\mathrm{ca} 1 \mu \mathrm{m})$, numerosos $(2 \mathrm{n}=52,56,104$ e 112$)$ e pouco estudados, sendo as informações disponíveis restritas a algumas contagens cromossômicas e análises meióticas.(GONZALEZ et al., 1967; PAN \& BREWBAKER, 1988; PALOMINO et al., 1995). Muitas das contagens não estavam confirmadas ou apresentavam dúvidas devido à falta de uma delimitação taxonômica bem definida.

Neste trabalho, são revistos e discutidos os resultados mais relevantes dos estudos citogenéticos em Leucaena realizados pelo grupo do Departamento de Plantas Forrageiras e Agrometeorologia da Universidade Federal do Rio Grande do Sul (DPFA), iniciados em 1984, e que compreendem: análises dos números cromossômicos, comportamento meiótico e fertilidade do pólen, em híbridos selecionados dentro de programas de melhoramento, e em espécies selvagens e cultivadas do gênero. Além disso, é comentada a contribuição destes trabalhos para o melhoramento genético e para o entendimento das relações citogenéticas entre as espécies do gênero.

\section{Citogenética de híbridos}

A análise de híbridos foi realizada a partir de materiais cruzados e selecionados pelo Dr. E. Mark Hutton no CIAT, Cali, Colômbia e CPAC-EMBRAPA, Planaltina,
Brasil, dentro de um programa de seleção de plantas para adaptação aos solos ácidos do cerrado brasileiro.

Foi estudado o número cromossômico e o comportamento meiótico em híbridos F1, F2 e F3 de $\boldsymbol{L}$. leucocephala $\times \boldsymbol{L}$. diversifolia, ambas $2 \mathrm{n}=104, \boldsymbol{L}$. leucocephala $\times$ L. diversifolia "diplóide" $(2 \mathrm{n}=52)$ [atual L. trichandra (Zucc.) Urban], L. leucocephala $x$ L. esculenta (Sessé \& Moc. ex DC.) Benth. $(2 n=52)$ e L. leucocephala $\times$ L. pulverulenta (Schltdl.) Benth. (FREITAS et al., 1988). Foi verificada uma grande variação interespecífica em árvores consideradas "puras" (L. leucocephala, L. diversifolia e $L$. trichandra) o que talvez possa ser explicado pela facilidade de hibridação natural entre estes taxas. Nos híbridos $\mathrm{F} 1$ entre estas espécies, os números eram muito variáveis, mas, nas $\mathrm{F} 2$ e F3, houve uma tendência a números mais altos, próximos do nível tetraplóide, indicando uma tendência ao aumento de números cromossômicos em gerações avançadas destas plantas selecionadas para tolerância a solos ácidos. Apenas bivalentes foram observados na meiose, o que levou os autores a sugerir a ocorrência de pareamento intraespecífico.

Trabalho posterior foi realizado com uma população de híbridos F2, F3 e F4 entre as tetraplóides L. leucocephala $\times$ L. diversifolia (Figura 2) (FREITAS et al., 1991). Para alguns caracteres morfológicos como número de flores por inflorescência e número de legumes por inflorescência, e cor da inflorescência, a população híbrida apresentou-se mais próxima a $\boldsymbol{L}$. leucocephala. Das 183 plantas analisadas, a grande maioria apresentava $2 \mathrm{n}=104$, mas alguns eventuais números mais baixos (86-98) foram observados em algumas árvores. Apesar de predominarem associações

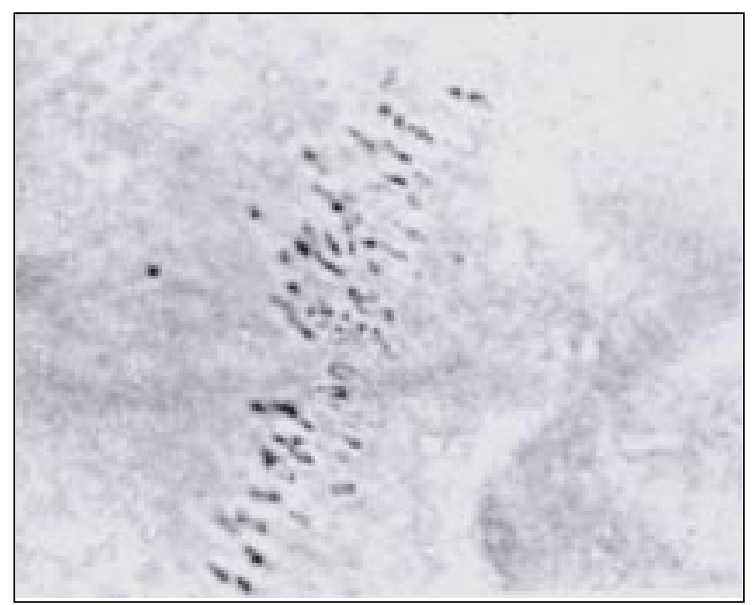

Figura 2 - Metáfase meiótica, 52II, em híbridos de Leucaena leucocephala e L. diversifolia; escala igual a $10 \mu \mathrm{m}$ 
em bivalentes (Figura 2), quadrivalentes trivalentes e univalentes foram observados. $\mathrm{O}$ indice meiótico, ou seja, a percentagem de tetrades de pólen normais, que reflete a estabilidade meiótica de uma planta, sendo consideradas estáveis aquelas com índices maiores ou iguais a $90 \%$, variou de 31,4 a $96,6 \%$ e a fertilidade do pólen de 14,8 a $96,2 \%$. Esta grande variabilidade indica a necessidade de um levantamento citogenético prévio para a seleção de genitores em cruzamentos controlados.

\section{Número cromossômico}

Dentro de uma linha ligada à taxonomia e evolução, os trabalhos realizados com acessos das diversas espécies, coletadas nos seus locais de ocorrência natural, foi, sem dúvida, a maior contribuição do grupo do grupo de citogenética.

Esta abordagem partiu de um intercâmbio com os pesquisadores do Oxford Forestry Institute (OFI), Universidade de Oxford, Reino Unido, Dr. Colin Hughes e Allan Pottinger, que disponibilizaram os acessos da coleção de germoplasma de Leucaena do OFI para análise citogenética.
A análise do número cromossômico de 73 populações das 22 espécies (SCHIFINO-WITTMANN et al., 2000; CARDOSO et al., 2000) (Tabela 1) mostrou resultados interessantes:

a) a descoberta de mais uma espécie tetraplóide no gênero, L. involucrata $\mathrm{S}$. Zárate, $2 \mathrm{n}=$ 112 ; b) a confirmação de contagens consideradas duvidosas; c) contagens inéditas para algumas espécies, como $L$. cuspidata Standley $(2 \mathrm{n}=52), \boldsymbol{L}$. lempirana C. E. Hughes (2n=52 e 56) (Figura 3), $\boldsymbol{L}$. magnifica (C. E. Hughes) C.E. Hughes $(2 n=52), \boldsymbol{L}$. matudae (S. Zárate) C.E. Hughes $(2 n=56), \quad \boldsymbol{L}$. pueblana Britton \& Rose $(2 \mathrm{n}=52)$; d) identificação de variabilidade intraespecífica em diversos taxa, como L. macrophylla Benth. subsp. istmensis $(2 \mathrm{n}=52$ e 56), L. confertiflora $\mathrm{S}$. Zárate var. adenotheloidea $(2 \mathrm{n}=104$ e 112), L. pallida $(2 \mathrm{n}=104$ e 112) and L. trichandra $(2 n=52$ and 104), em alguns casos inclusive aparentemente ligada à delimitação taxonômica de subespécies (L. collinsii Britton \& Rose subsp. collinsii $2 \mathrm{n}=52$, subsp. zacapana $2 \mathrm{n}=56, \boldsymbol{L}$. macrophylla Benth. subsp. istmensis $2 \mathrm{n}=52,56$, subsp. macrophylla $2 \mathrm{n}=56$ ).

Tabela 1 - Números cromossômicos em Leucaena obtidos na literatura : Hughes (1998 b) e Schifino-Wittmann et al. (2000) e Cardoso et al. (2000).

\begin{tabular}{|c|c|c|}
\hline Espécies & 2n (Hughes1998) & 2n (Schifino-Wittmann et al., 2000 e Cardoso et al., 2000) \\
\hline L. collinsii Britton \& Rose & $52(? 56)$ & 52 and $56^{*}$ \\
\hline L. confertiflora S. Zárate & 112 & $104 *$ and 112 \\
\hline L. cuspidata Standley & $?$ & $52 *$ \\
\hline L. diversifolia (Schltdl.) Benth. & 104 & 104 \\
\hline L. esculenta (Sessé \& Moc. ex DC.) Benth. & $52(? 56)(? 112)$ & 52 \\
\hline L. greggii S.Watson & 56 & 56 \\
\hline L. involucrata S.Zárate & $?$ & $112 *$ \\
\hline L. lanceolata S. Watson & 52 & 52 \\
\hline L. lempirana C.E. Hughes & $?$ & $52^{*}, 56^{*}$ \\
\hline L. leucocephala Lam. (de Wit) & 104 & 104 \\
\hline L. macrophylla Benth & $? 52$ & $52 *$ and $56^{*}$ \\
\hline L. magnifica (C.E.Hughes) C.E.Hughes & $? 52$ & $52 *$ \\
\hline L. matudae (S. Zárate) C.E.Hughes & $? 52$ & $56^{*}$ \\
\hline L. multicapitula Schery & $? 52$ & $52 * *$ \\
\hline L. pallida Britton \& Rose & $104(? 110,112)$ & 104 and $112 *$ \\
\hline L. pueblana Britton \& Rose & $?$ & $52 *$ \\
\hline L. pulverulenta (Schltdl.) Benth. & 56 & 56 \\
\hline Espécies & 2n (Hughes1998) & 2n (Schifino-Wittmann et al., 2000 e Cardoso et al., 2000) \\
\hline L. retusa Benth. & 56 & $52 *$ \\
\hline L. salvadorensis Standley ex Britton \& Rose & $? 56$ & $56^{* *}$ \\
\hline L. shanonii J.D. Smith & 52 & $52,56 * *$ \\
\hline L. trichandra (Zucc.) Urban & $52(? 56)$ & 52 and $104 *$ \\
\hline L. trichodes (Jacq.) Benth & 52 & $56^{*}$ \\
\hline
\end{tabular}

* contagens novas

** confirmação de contagens controversas 
Os dados de número cromossômico, em síntese, demonstraram a grande variabilidade, mesmo em números básicos [n=26 e 28 considerando-se as espécies supostamente diplóides do gênero, ou $\mathrm{x}=13$ e 14 considerando-se outras Mimosoideae (GOLDBLATT, 1981)] nas espécies de Leucaena. Além disto, os resultados sugerem: a) que as diferenças interespecíficas de número cromossômico poderiam ter surgido por uma provável disploidia nas espécies diplóides e consequentemente nas tetraplóides; b) origens múltiplas das espécies tetraplóides; e apoiam o padrão evolutivo complexo do gênero (evolução reticulada) como sugerido por HUGHES \& HARRIS (1995) e HARRIS et al. (1996).

\section{Comportamento meiótico}

Uma análise recente do comportamento meiótico e fertilidade do pólen em 49 acessos de 14 taxa de Leucaena (BOFF \& SCHIFINO-WITTMANN, 2002; BOFF \& SCHIFINO-WITTMANN, 2003) (Tabela 2) mostrou que as espécies diplóides L. pulverulenta, L. retusa Benth., L. shannonii J.D. Smith e $\boldsymbol{L}$. trichandra apresentaram comportamento meiótico preferencialmente em bivalentes e quadrivalentes. Foram também observados univalentes e outras associações múltiplas, porém em freqüências variadas. O acesso estudado de $\boldsymbol{L}$. macrophylla apresentou predominância de células com anormalidades. Nas espécies tetraplóides $L$. confertiflora, L. diversifolia, L. involucrata, L. leucocephala (Figura 3), L. pallida e L. $\boldsymbol{x}$ spontanea (Tabela 2), apesar da predominância

Tabela 2 - Associações cromossômicas em espécies diplóides e tetraplóides de Leucaena (adaptado de Boff \& SchifinoWittmann, 2002; Boff \& Schifino-Wittmann, no prelo)

\begin{tabular}{lcccc}
\hline Espécie & $2 \mathrm{n}$ & $\begin{array}{c}\mathrm{n}^{\mathrm{o}} \\
\text { acessos }\end{array}$ & $\begin{array}{c}\text { associações } \\
\text { cromossômicas }\end{array}$ \\
\hline L. macrophylla & 56 & 1 & $28 \mathrm{II}$ & $(98,0) *$ \\
L. pulverulenta & 56 & 3 & $28 \mathrm{II}$ & $(16,0)$ \\
L. retusa & 52 & 1 & $26 \mathrm{II}$ & $(39,0)$ \\
L. shannonii & 52 & 2 & 26 II & $(48,0)$ \\
& 56 & 1 & $28 \mathrm{II}$ & $(60,0)$ \\
L. trichandra & 52 & 7 & 26 II & $(7,0)$ \\
L. confertiflora & 104 & 1 & 52 II & $(21,7)$ \\
L. diversifolia & 104 & 9 & $52 \mathrm{II}$ & $(9,0)$ \\
L. involucrata & 112 & 1 & 56 II & $(55,0)$ \\
L. leucocephala glabrata & 104 & 11 & $52 \mathrm{II}$ & $(17,0)$ \\
L. leucocephala leucocephala & 104 & 1 & $52 \mathrm{II}$ & $(55,0)$ \\
L. pallida & 112 & 2 & 56 II & $(43,0)$ \\
Lx spontanea & 104 & 1 & $52 \mathrm{II}$ & $(51,0)$ \\
\hline
\end{tabular}

*entre parênteses, percentagem máxima de células com irregularidades, principalmente univalentes, trivalentes, quadrivalentes, outros multivalentes

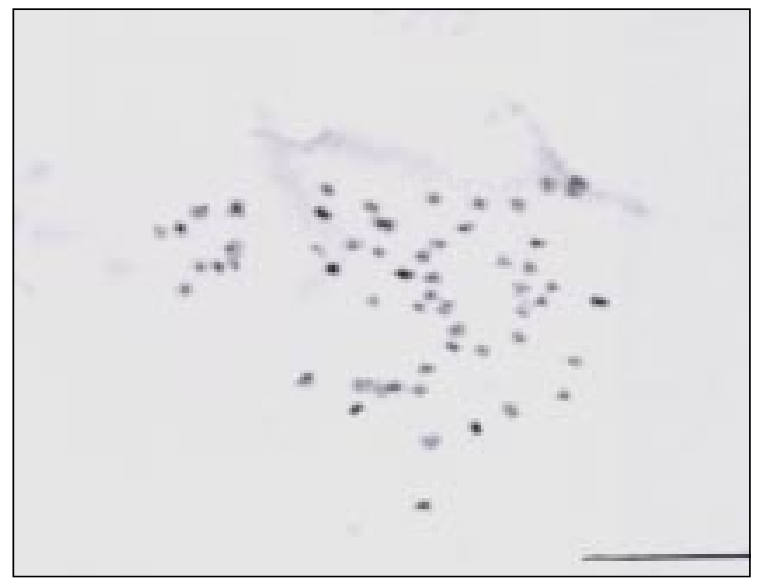

Figura $3-$ L. lempirana, $2 \mathrm{n}=56$; escala $10 \mu \mathrm{m}$

de bivalentes, quadrivalentes e outras irregularidades foram observadas em frequiências variadas.

Os índices meióticos médios e a fertilidade média do pólen, incluindo também $L$. salvadorensis Standley ex Britton \& Rose e L. cuspidata, variaram de 73,5 a $98,9 \%$ e de 54,2 a $98,8 \%$, respectivamente.

Estes dados sugerem que as espécies diplóides de Leucaena podem ser paleopoliplóides, como já proposto com base no alto número cromossômico 'diplóide' ( 2 n=52 e 56) do gênero, quando comparado com os números básicos sugeridos para algumas Mimosoideae ( $\mathrm{x}=13$ e 14) (GOLDBLATT, 1981). Em relação às espécies poliplóides, dados da literatura (HUGHES, 1998 a) têm proposto origem alopoliplóide. Os dados de comportamento meiótico sugerem uma origem alopoliplóide segmentar sensu STEBBINS (1971), apesar de a autopoliploidia não poder ser descartada. Até 7\% (BOFF \& SCHIFINO-WITTMANN, 2003) ou mesmo 12\% (SCHIFINO-WITTMANN et al., 2000) de gametas não reduzidos podem ocorrer em $\boldsymbol{L}$. trichandra. A identificação de um poliplóide natural em $\boldsymbol{L}$. trichandra (SCHIFINO-WITTMANN et al., 2000) sugere que a autopoliploidia pode ocorrer em Leucaena. Dados recentes de DNA de cloroplastos (cp DNA), e de espaçadores gênicos internos (ITS) (HUGHES et al., 2002) sugerem uma origem alotetraplóide para quatro das espécies tetraplóides de Leucaena (nas quais foi detectado polimorfismo para ITS) mas não excluem a possibilidade de autotetraploidia em $\boldsymbol{L}$. diversifolia, já que nesta última apenas um tipo de ITS foi encontrado (Figura 4).

Em resumo, os trabalhos aqui discutidos em termos de número de cromossomos em Leucaena revelam variabilidade intra e interespecífica, 


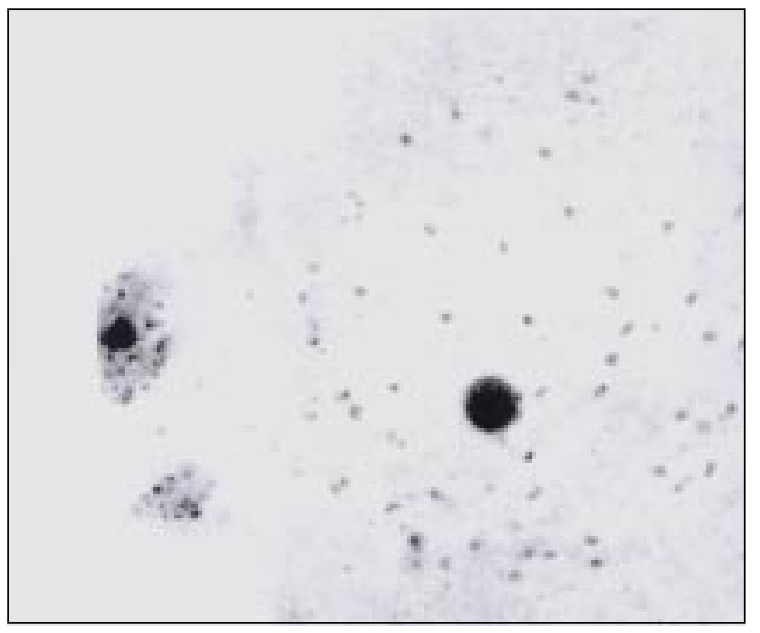

Figura 4 - Diacinese em L. leucocephala glabrata, 52 II ; escala $10 \mu \mathrm{m}$

demonstram a ocorrência de multivalentes, mesmo nas espécies consideradas diplóides, apoiando a origem paleopoliplóide de muitas espécies e constituem uma importante contribuição para a citogenética destas espécies. Estes resultados também podem trazer repercussões na taxonomia e no melhor entendimento da complexa evolução do gênero. Além disto, demonstram a importância de um acompanhamento citogenético em programas de melhoramento genético destas espécies.

\section{REFERÊNCIAS BIBLIOGRÁFICAS}

BOFF, T.; SCHIFINO-WITTMANN, M.T. Pollen fertility and meiotic behaviour in accessions and species of Leucaena. Tropical Grassland, Brisbane, v.36, p.54-58, 2002

BOFF, T.; SCHIFINO-WITTMANN, M.T. Paleopolyploidy and segmental allopolyploidy in species of Leucaena Benth evidence from meiotic behaviour analysis. Hereditas, Lund, v. 138, p. $27-35,2003$.

BREWBAKER, J.L. Species in the genus Leucaena. Leucaena Research Reports, Taiwan, v.7, p.6-20, 1987.

CARDOSO, M.B.; SCHIFINO-WITTMANN, M.T.; BODANESE-ZANETTINNI, M.H. Taxonomic and evolutionary implications of intraspecific variability in chromosome numbers of species of Leucaena Benth (Leguminosae). Botanical Journal of the Linnean Society, London, v.134, p.549-556, 2000.

FREITAS, L.H.C.; SCHIFINO-WITTMANN, M.T.; HUTTON, E.M. Cytogenetic analysis of species and hybrids of Leucaena (Leguminosae) in relation to acid soil tolerance. Brazilian Journal of Genetics, Ribeirão Preto, v.11, p.97109,1988
FREITAS, L.H.C.; SCHIFINO-WITTMANN, M.T.; PAIM, N.R. Floral characteristics, chromosome numbers and meiotic behavior of hybrids between Leucaena leucocephala $(2 \mathrm{n}=104)$ and tetraploid L. diversifolia $(2 n=104)$ (Leguminosae). Brazilian Journal of Genetics, Ribeirão Preto, v.14, p.781-789, 1991.

GOLDBLATT, P. Cytology and the phylogeny of the Leguminosae. In: POLHILL, R. M.; RAVEN, P.H. (Eds). Advances in legume systematics. Part 2. Kew : Royal Botanic Gardens, 1981. p.427-464.

GONZALEZ, V.; BREWBAKER, J.L.; HAMIL, D.E. Leucaena cytogenetics in relation to the breeding of low mimosine lines. Crop Science, Madison, v.7, p.140-143, 1967.

HARRIS, S.A.; CHAMBERLAIN , J. R.; HUGHES C.E. New insights into the evolution of Leucaena Benth. In: PICKERSGIL, B.; LOCK, J.M. (eds.). Advances in legume systematics. Part 8. Legumes of economic importance. Kew : Royal Botanic Gardens, 1996. p.117-126.

HUGHES, C.E. Leucaena genetic resources: The OFI seed collections and a synopsis of species characteristics. Oxford : Oxford Forestry Institute, 1993. $117 \mathrm{p}$.

HUGHES, C.E. Monograph of Leucaena (LeguminosaeMimosoideae). Systematic botany monographs 55. Ann Harbor : The American Society of Plant Taxonomists, 1998a. $244 \mathrm{p}$.

HUGHES, C.E. Leucaena. A genetic resources handbook. Tropical forestry paper 37. Oxford : Oxford Forestry Institute, 1998b. 274p.

HUGHES, C.E.; HARRIS, S.A. Systematics of Leucaena: recent findings and implications for breeding and conservation. In: SHELTON, H.M.; PIGGIN, C.M.; BREWBAKER, J.L. (eds.). Leucaena- opportunities and limitations. ACIAR Proceedings 57. Canberra: ACIAR, 1995. p.54-65.

HUGHES, C.E.; BAILEY, C.D.; HARRIS, S.A. Divergent and reticulate species relationships in Leucaena (Fabaceae) inferred from multiple data sources: insights into polyploid origins and nrDNA polymorphism. American Journal of Botany, Ithaca, v.89, p.1057-1073, 2002.

PALOMINO, G.; ROMO, V.; ZÁRATE S. Chromosome numbers and DNA content in some taxa of Leucaena (Fabaceae Mimosoideae). Cytologia, Tokyo, v.60, p.31-37, 1995.

PAN, F.J.; BREWBAKER, J.L. Cytological studies in the genus Leucaena Benth. Cytologia, Tokyo, v.53, p.393399, 1988.

SCHIFINO-WITTMANN, M.T. Hybrids between Leucaena leucocephala and L. diversifolia in Rio Grande do Sul, Southern Brazil; a summary. Leucnet News, Oxford, v.7, p.3-15, 2000 .

SCHIFINO-WITTMANN, M.T. et al. Chromosome numbers and unreduced gametes in species of Leucaena Benth (Leguminosae) - new contributions for the taxonomy, evolutionary studies and breeding of the genus. In: GUTtenBERGER, H. et al. (eds.) Cytogenetic 
studies of forest trees and shrubs - Review, present status and outlook on the future. Zvolen: Arbora , 2000.p.181-190

STEBBINS, G.L. Chromosomal evolution in higher plants. Reading: Addison-Wesley, 1971. 216 p.

ZÁRATE, S. Revisión del genero Leucaena in Mexico. Anales del Instituto de Biologia, serie Botanica, Mexico v. 65, p. 83-162, 1994.

Ciência Rural, v.34, n.1, jan-fev, 2004. 